\title{
Wide bandwidth directional beaming via waveguide ports in photonic crystals
}

\section{Zhaofeng Li, Koray Aydin and Ekmel Ozbay}

Nanotechnology Research Center, Department of Physics, and Department of Electrical and Electronics Engineering, Bilkent University, Bilkent, 06800 Ankara, Turkey

E-mail: zhaofengli@bilkent.edu.tr

Received 4 March 2008, in final form 3 June 2008

Published 18 July 2008

Online at stacks.iop.org/JPhysD/41/155115

\begin{abstract}
We numerically and experimentally demonstrated highly directional emission from photonic crystals (PCs). Our structures comprise branched waveguide channels with a periodic doubling scheme. An incident electromagnetic wave can be first split into multiple beams using PC waveguide structures. The beams were then emitted at the output waveguide ports of the PC with the same phase, which resulted in a highly directional radiation pattern. Our results demonstrate that the full width at half maximum of the emission beam is nearly inversely proportional to the number of output waveguide ports. Typically, for a PC with sixteen output ports, the half-power beam width measured $4.8^{\circ}$, which was in good agreement with the calculated value of $4.1^{\circ}$. In contrast to the traditional beaming structures, our design did not involve grating-like structures, which resulted in a wider operation bandwidth.
\end{abstract}

(Some figures in this article are in colour only in the electronic version)

M Animation files for figure 3 are available in the online edition. 


\section{Introduction}

It is well known that light waves will be diffracted into a wide angle after they transmit through a subwavelength aperture. This characteristic sets a lower limit on the integration density of many optical devices. In 2002, Lezec et al demonstrated an exotic phenomenon wherein an enormously enhanced transmission and beaming emission was obtained when a light wave was propagated through and out of a subwavelength metallic aperture [1]. They achieved these results by surrounding the subwavelength aperture with periodic textures on the input and output sides. It was then thought that the excitation of the surface wave, or the surface plasmons, on the metallic surfaces plays the dominant role in this phenomenon [2-5]. Following the pioneer works of Lezec et $a l$ there has been much effort put forth on the study of the enhanced transmission and beaming effect (or directional emission)

electromagnetic(EM)wavesthroughametallicsubwavelength aperture [6-8].

Intrigued by these more than abnormal phenomena, not muchlateronitwasfoundthatthebeamingeffectcouldalsobe obtained in photonic crystals (PCs) $[9,10]$. PCs are materials that comprise periodical dielectric distributions. Due to the existence of photonic band gaps, PCs can be used to control 0022-3727/08/155115+06\$30.00

only within a relatively narrow bandwidth, which is controlled by the period of the covering grating-like layer or band edges.

As stated in the previous paragraph, one of the special properties of PCs are their ability to manipulate the flow of light waves on a wavelength scale. Many applications are based on this property, such as bending [19] and splitting [20]. In a recent work [21], we reported a design for highly directional emission from a PC waveguide wherein the bending and splitting abilities of a PC waveguide were used. Except for these two mechanisms, no grating-like structure was adopted in our design. While it is the gratinglike structure that intrinsically limits the operating bandwidth of previous designs, our structure can possess a wide operating bandwidth. However, only one special design (with 16 waveguide ports) is presented in our former short letter [21], which is not enough to show the efficiency and flexibility of our design method. In this work, we demonstrate directional beaming for a series of designs. Consequently, we can further investigate the relation between the emitting surface width and the full width at half maximum (FWHM) of the emitting beam. In previous designs that used grating-like structures, althoughitwasdemonstratedthatalongwiththeincreaseinthe emitting surface width the FWHM will decrease accordingly, no detailed quantitative description was provided. However, for our design, we will show that the FWHM of the emitting beamhasapredictablerelationwiththeemittingsurfacewidth, which can be described quantitatively. The following sections are arranged as follows. In section 2, we illustrate the PC structure under study and the detailed designs of a series of beaming structures. In section 3, we show the simulation and the propagation of light waves on the scale of a wavelength, in which this characteristic makes PCs a promising candidate as the building blocks of future integrated optical circuits [11]. It was first demonstrated that directional emission could be realized by properly selecting the termination surface for the PC waveguide [9]. Meanwhile, a more general method for obtaining a beaming effect was proposed, in which a surface mode and a grating-like layer was suggested to be added to the emitting surface of the PC waveguide [10,12,13]. All the above beaming effects that occurred in PCs are based on the excitation of the surface modes in the exit PC waveguide face, in which these surface modes are then diffracted by the grating-like layer and transformed into a directional beaming. Moreover, it is also reported that by covering the termination of the PC waveguide with a self-collimation PC [14], cavities [15], or even a coupled cavity PC waveguide [16], directional emission can also be achieved. It is well known that PCs can not only have photonic band gaps but also exotic dispersion characteristics in the band structure. By taking advantage of the dispersion properties, directional emission can also be obtained. For instance, by placing a radiation source inside the PC, a beaming effect can be obtained at the frequency of the band edge [17,18]. Although these above-reported beaming phenomena come from different mechanisms, they all work

(C) 2008 IOP Publishing Ltd Printed in the UK

experimental results of the directional emission, while discussions are located in section 4. Conclusions are presented in section 5 .

\section{The PC and beaming structure}

For simplicity, a two-dimensional (2D) PC was employed in our study. The inset of figure 1(a) shows the schematic of the PC structure, while figure 1(a) shows the band structure. The $\mathrm{PC}$ is a 2D square lattice of alumina rods embedded in air. The alumina rods have a refractive index of 3.1, a radius of $\mathrm{r}=$ $1.575 \mathrm{~mm}$, and the lattice constant a is $11 \mathrm{~mm}$. As shown in figure 1(a), this PC has a band gap for transverse magnetic (TM) polarization (with magnetic fields perpendicular to the axis of the rods) between band 0 and band 1 . In fact, our calculation shows that this band gap has the frequency range $0.376-0.482 \mathrm{a} / \lambda$, where $\lambda$ is the wavelength in free space. This frequency range also corresponds to $10.26-13.15 \mathrm{GHz}$ for the experimental structure. A PC waveguide can be formed after onecolumnofrodsisremoved, inwhichtheinsetoffigure1(b) shows the schematic of the PC waveguide. This PC waveguide is highly dispersive as can be seen from the calculated results shown in figure 1(b).

In order to obtain a beaming emission from the exit of a PC waveguide, we designed a PC structure that has branched waveguide channels with a periodic doubling scheme. Figure 2(a) shows a PC waveguide structure with its endopeneddirectlyonthesurfaceofthePC, whichwillbeused 

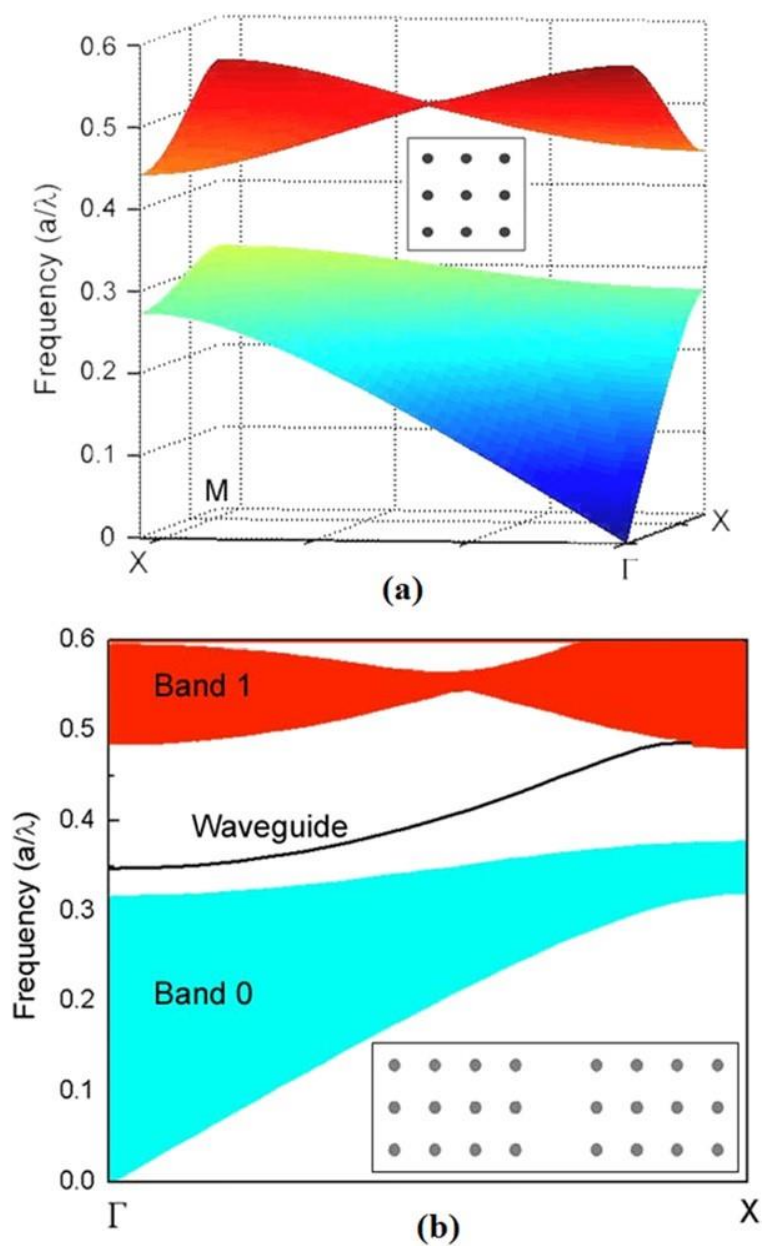

Figure 1. (a) The band structure (TM polarization) of the 2D PC. The inset shows the PC structure with a square lattice. The black dots represent the dielectric rods (with refractive index 3.1) placed in air. (b) The projected band structure of the PC waveguide, which is formed by removing one column of the rods from the $\mathrm{PC}$, is shown in the inset. The shaded regions are the projected pass bands, while the solid line is the dispersion curve of the PC waveguide mode.

as a reference for other beaming structures. Figures 2(b)-(d) are three designed PC structures for directional beaming. Amongthem, theoutputpartofthePCinfigure2(b)consistsof four PC waveguide ports, while the PC structures in figure 2(c) and (d) have eight and sixteen output ports, respectively. It can be clearly seen in figure 2(b) that when a beam of an EM wave propagates from the bottom to the top, it will be split into four beams when they reach the output ports. As all four of the beams propagate the same length, they will then have the samephasewhentheyarriveattheoutputports. Consequently, these waves are expected to form good directional emission. This mechanism should also work for the PC structures shown in figures 2(c) and (d). Moreover, along with an increase in the waveguide port number, the directivity of the output beam should also improve.

\section{Simulation and experimental results}

To study the possible beaming effect that we expected in the former section, 2D finite-difference time-domain (FDTD)

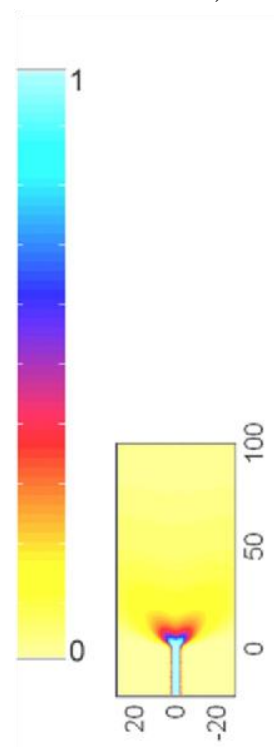

(a)

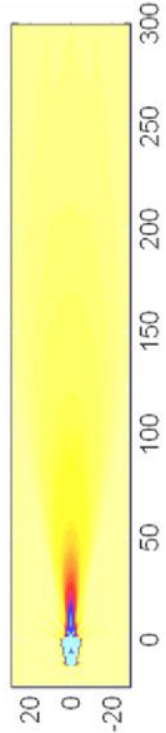

(b)

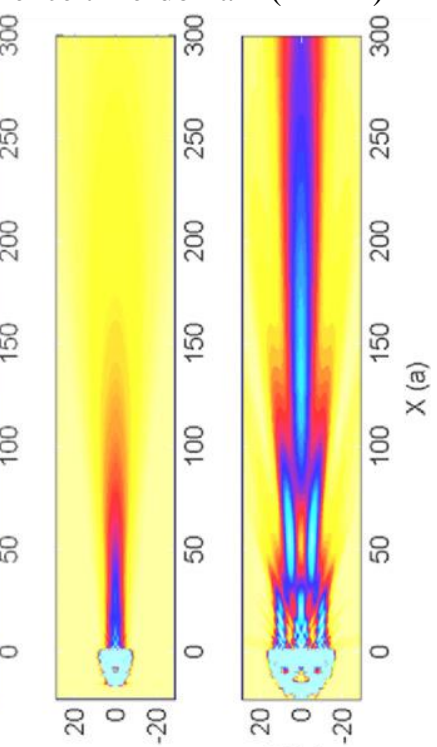

(c)

(d) $Y(a)$ 


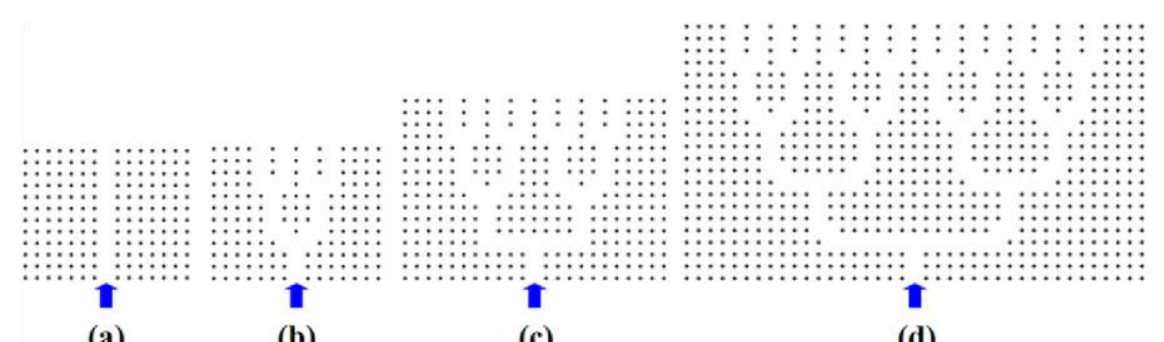

(a)

(b)

(c)

(d)

Figure 2. (a) The PC structure where the output port of waveguide is opened directly on the PC surface. (b), (c) and (d) show three proposed PC beaming structures wherein a single waveguide port is split into 4,8 and 16 output ports, respectively.

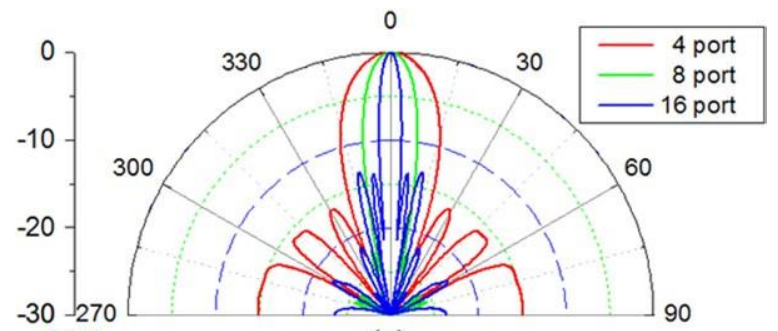

$(\mathrm{dB})$

(a)

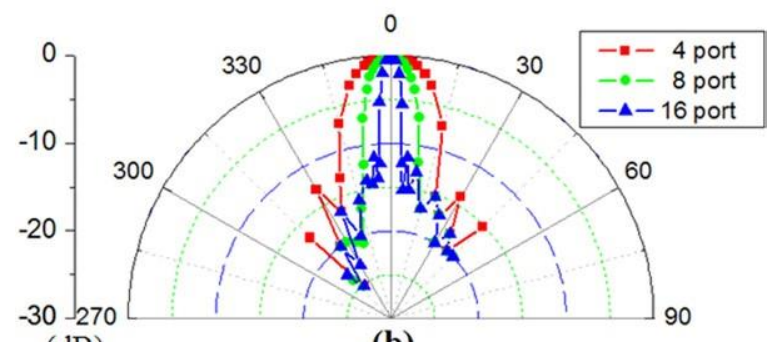

(dB)

(b)

Figure 3. (a) The intensity distributions of the electric field for the line defect structure as shown in figure 2(a). Figures (b), (c) and (d) show the intensity distributions of the electric field for the structures shown in figure 2 with 4,8 and 16 output ports, respectively. All the simulations were performed at a frequency of $\mathrm{f}$ $=0.417 \mathrm{a} / \lambda(11.36 \mathrm{GHz})$, and the $\mathrm{X}$ and $\mathrm{Y}$ axes were scaled in the PC lattice constant a. Supplementary files presenting animations of the wave propagation in parts (b), (c) and (d) are available in the online edition.

[22] simulations were carried out for the structures shown in figure 2. In the simulation, the perfect matched layers [23] are applied as absorption boundaries. In the simulations, the frequency was $f=0.417 \mathrm{a} / \lambda$ (equivalent to $11.36 \mathrm{GHz}$ ), which is located near the middle of the PC waveguide mode bandasshowninfigure1(b). Inthesimulation, theinputwaves are launched into the bottom ports of the beaming structures as shown in figure 2. After the EM waves emit from the upper ports, theirintensitydistributionsarerecorded. Figure 3 shows the recorded field intensity distributions for the three beaming structures and the reference structure. Figure 3(a) shows the result of the reference structure, from which it can be seen that the emitted EM wave is diffracted into a wide angle just as expected. Figures 3(b), (c) and (d) show the simulation results for the beaming structures of figures 2(b), (c) and (d). It can be clearly seen that our beaming structures really work well in forming beaming emissions. Furthermore, along with the increase in the number of output waveguide ports, the beaming

Figure 4. (a) The calculated far field radiation patterns for the three beaming structures with 4,8 and 16 output ports, respectively. The calculated FWHM of the three structures are $18.9^{\circ}, 10.5^{\circ}$ and $4.1^{\circ}$, respectively. (b) The measured far field results of the corresponding three beaming structures. The measured FWHM of the three structures are $20.1^{\circ}, 10.9^{\circ}$ and $4.8^{\circ}$, respectively. All the above results are obtained for a frequency of $\mathrm{f}=0.417 \mathrm{a} / \lambda(11.36 \mathrm{GHz})$.

emission is confined to a much narrower angle. Especially for the beaming structure with sixteen output waveguide ports, the emitted EM wave beam possesses exceptional directivity.

Since we obtained qualitative beaming effects, we wanted to calculate the far field patterns for a better evaluation of the performance of our structures. The frequency of the calculation was set at $\mathrm{f}=0.417 \mathrm{a} / \lambda$ which is the same as the frequency for the intensity distribution results as shown in figure 3. During the calculation of far field radiation patterns, Kirchoff's integral was applied to transform near field data into far field data. Figure 4(a) shows the calculated results of the far field radiation patterns for the three beaming structures, and the FWHM data for the three beaming structures with 4, 8 and 16 output ports at $18.9^{\circ}, 10.5^{\circ}$ and $4.1^{\circ}$, respectively. It can be seen clearly that all the structures emit beaming emissions. Moreover, along with an increase in the number of the output waveguide ports, the directivity (measured by the FWHM) of the emission beam improves markedly. Typically, 
when the number of output waveguide ports reaches sixteen, the emission beam from the PC structure possesses excellent directivity (with FWHM of only $4.1^{\circ}$ ).

In order to validate our simulation results, we conducted experiments in the microwave regime. In the experiments, two horn antennas were used to connect to an HP-8510C network analyzer. One horn antenna was excited in order to obtain an incident EM field [12], while the other horn antenna acted as a receiver that measured the EM power that was emitted from the output ports of the PC structure. The length of the alumina rods used to construct the PC structure was $15 \mathrm{~cm}$, which is at least five times longer than the EM wavelengths used in this study. This configuration made the constructed PC structure a good approximation to the structure that was used in the $2 \mathrm{D}$ FDTD simulations. In the measurement of the far field radiation patterns, the receiver horn antenna was set to scan a semi-circle with the radius $\mathrm{R}=1.8 \mathrm{~m}$ from the centre of the exit face of the PC structure. The measured results of the far field radiation patterns for the three beaming structures are shown in figure 4(b), and the measured FWHM for the three beaming structures with 4,8 and 16 output ports were $20.1^{\circ}, 10.9^{\circ}$ and $4.8^{\circ}$, respectively. The measured FWHM results are slightly larger than the calculated ones, which might be partially due to the imperfections in the constructed structure, e.g. the slight randomness in the lattice constant, and the radii ofthedielectricrods. Inaddition, thephysicalwidthofthehorn antennaandsomeuncertainty $\left( \pm 2^{\circ}\right)$ initsexactorientationcan also affect the accuracy of the measurement. Nevertheless, our experimental results are in quite good agreement with the simulation results.

As was pointed out in the first section, since most of the PC based beaming structures depend on grating-like structures, the intrinsic property of the grating will limit the beaming effect into a narrow operating frequency bandwidth. However, in our beaming structures, only PC waveguides are used to split and then combine these EM beams in order to achieve highly directional radiation. Consequently, it is expected that our beaming structures would have a wider operation frequency bandwidth when compared with the grating-like structure based PC beaming structures. In order to validate this characteristic, we calculated and measured the far field radiation patterns for the beaming structure within a wide frequency bandwidth. It is noteworthy that although the PC waveguide has a working frequency band within 0.376$0.482 \mathrm{a} / \lambda$ (as shown figure $1(\mathrm{~b})$ ), the group velocity of the waveguide mode becomes increasingly slow when approaching the band edges. The very slow group velocity makes the incident wave rather difficult to couple into the input port of the PC waveguide. After considering this factor, we limited our calculation and experimental study within the frequency range $0.40-0.47 \mathrm{a} / \lambda$.

The calculated FWHM data of the far field radiation patternsforthePCbeamingstructuresareshowninfigure5(a). It can be clearly seen that all the three beaming structures keep up a good performance within the whole frequency range of interest. In fact, this property is further verified by our experimental results as shown in figure 5(b). Similar to the situation of figure 4, the measured FWHM data are slightly larger than the calculated results. After considering the uncertain factors in the measurement as we stated in the previous paragraphs, the experimental and simulation results
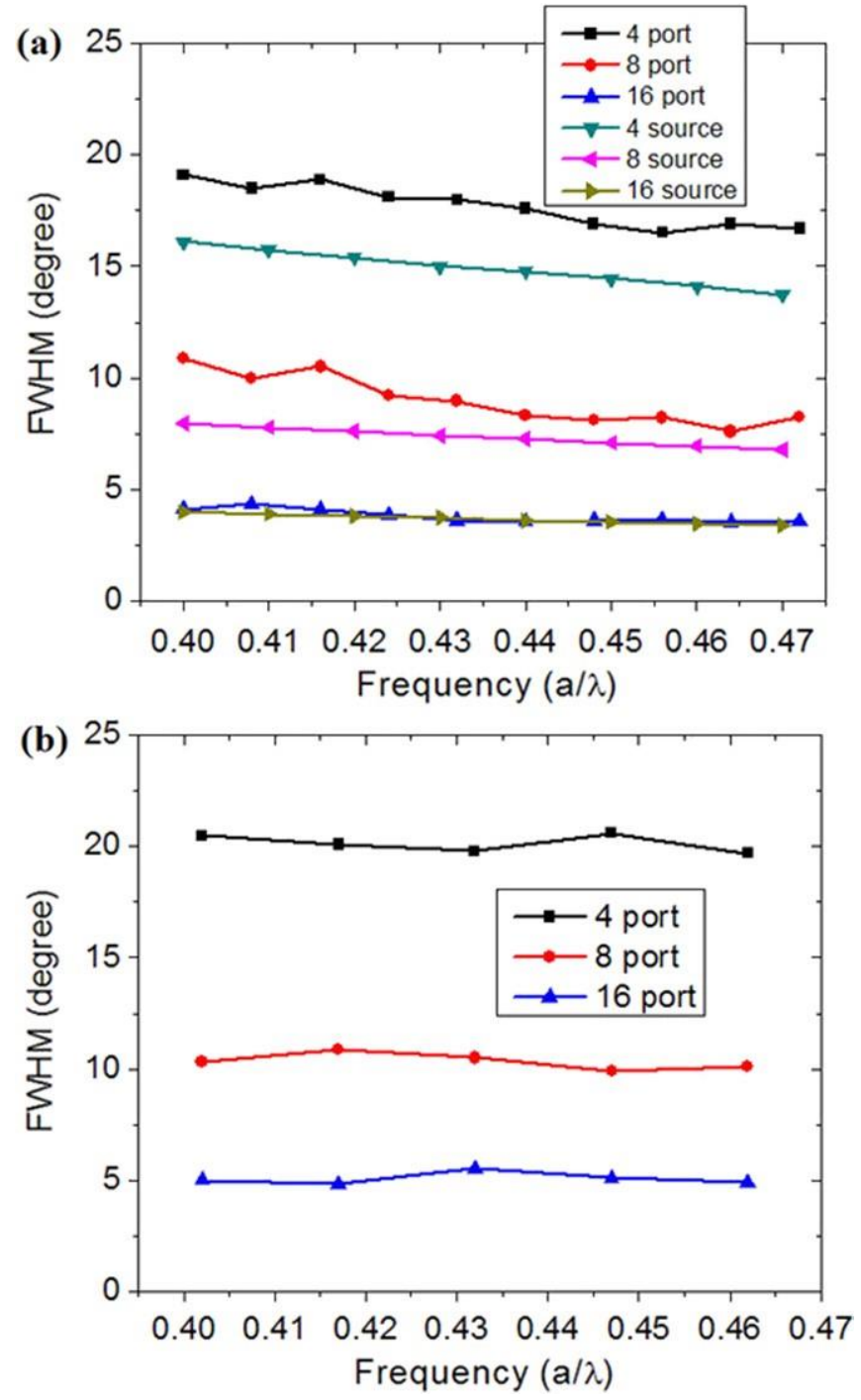

Figure 5. (a) The FWHM data of the calculated radiation patterns for the beaming structures with 4,8 and 16 output ports within the frequency range $0.40-0.47 \mathrm{a} / \lambda$. The FWHM data of the radiation patterns for 4, 8 and 16 coherent point sources are also shown for comparison purposes. (b) The measured FWHM data of the radiation patterns for the three beaming structures.

are in good agreement. Consequently, it is evident that our PC beaming structures can really work well over a wide frequency bandwidth.

\section{Some discussions}

In order to gain more information about the underlying mechanism that yields the beaming phenomenon, we also carried out FDTD simulations with barely coherent point 
sources. In the simulations, 4,8 and 16 coherent point sources were placed at the centres of the output waveguide channels of the corresponding PC beaming structures, but the real PC structures were removed. The FWHM of the emission beams from three different numbers of coherent point sources are also illustrated as a function of frequency in figure 5(a). By comparing the FWHM data from the PC beaming structures with the results of the coherent point sources, it is easy to see

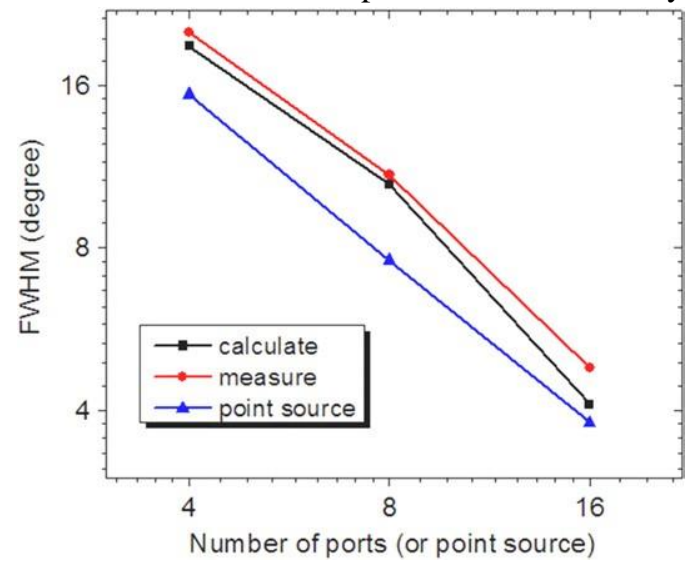

Figure 6. Comparison of the calculated and measured FWHM results for the three structures with a different port number at a frequency of $f=0.417 \mathrm{a} / \lambda(11.36 \mathrm{GHz})$.

that the results of 16 waveguide ports and 16 point sources are rather approximate, while the results of the 4 waveguide ports areobviouslylargerthanthatofthe4pointsources. Thereason for this may come from the emission profile of a single output waveguide port. As seen in figure 3(a), the emission profile of a single output waveguide port is different from a typical point source. For the beaming structure with only 4 output ports, this imperfect emission profile may have a large effect on the formation of a good quality emission beam. However, when the number of the output ports increases, the imperfection of each single emission profile will only have a slight effect on the final formation of a good quality emission beam. In this case, it is correct to state that the PC beaming structure with 16 output waveguide ports acts very similar to the 16 coherent point sources.

Now, let us discuss the relation between the FWHM of the emission beams and the number of output waveguide ports. For the traditional PC beaming structures that are based on the excitation of surface waves and the diffraction of a gratinglike structure, the field intensity on the emitting surface is very uneven. With the increase in the distance of the position on the emitting surface from the output PC waveguide port, the field intensity becomes increasingly lower. Consequently, only the emitting surface, which is near the output waveguide port, can have a significant contribution to the formation of beaming. Therefore, there is an upper limit for the best directivity that can be achieved for the PC beaming structures using a gratinglike structure. However, our $\mathrm{PC}$ beaming structure does not use a grating-like structure. All the output waveguide ports emit a nearly equal field intensity, which is very efficient for the formation of highly directive emission beams. Figure 6 plots the FWHM data as a function of the number of output waveguide ports and point sources. The scale of the $\mathrm{x}$ and $\mathrm{y}$ axes are set to be logarithm with its base, which is 2 . For the FWHM data of the coherent point sources, the FWHM data are perfectlyinverselyproportionaltothenumberofpointsources, which is consistent with the theoretical prediction. As for the three PC beaming structures, this inverse proportional relation was not very good. The reason is similar to the reason stated in the previous paragraph, which is that the emission profile of a single output waveguide port is different from a perfect point source. Nonetheless, it is still relevant to state that the FWHM of the emission beams are nearly inverse proportional to the number of output ports. This characteristic provides our proposed design with a predictable performance when we attempt to increase the number of output waveguide ports. Furthermore, theoretically speaking, there is no limitation on the minimum value of FWHM that can be achieved. In other words, the FWHM of the emission beam can be infinitely close to zero. This characteristic makes our design rather powerful.

We would also like to note that no optimization was applied for our beaming structure. Some special structures can be further added to the present structure in order to enhance the efficiency of the beam bending [24], beam splitting [25] and coupling of the output ports to free space [26]. Although a dielectric rod type PC is adopted here to demonstrate the performance of our design, the principle of our design can also work well for air hole type PCs. Besides, if the wave propagation direction is reversed, our designed structure can be used to effectively couple a wide beam of EM wave from a homogeneous medium into a narrow PC waveguide.

\section{Conclusions}

In conclusion, we designed a PC structure consisting of branchedwaveguidechannelswithaperiodicdoublingscheme in order to obtain directional emission. We experimentally and numerically achieved a highly directional emission from the designed PC structure. For structures with different numbers of output ports, the FWHM of the emitted beam is nearly inversely proportional to the number of output ports. This characteristic provides our design with a predictable performance. When compared with grating-like beaming structures, our PC beaming structure can operate over a wider bandwidth. Our design principle can be easily generalized to other types of PC structures. This design can be used in PC lasersandinotherapplicationsforachievinghighlydirectional emission. 


\section{Acknowledgments}

This work is supported by the European Union under theProjectsEU-NoE-METAMORPHOSE,EU-NoE-

PHOREMOST and TUBITAK and under Project Nos 105E066, 105A005, 106E198 and 106A017. One of the authors

(EO) also acknowledges the partial support from the Turkish Academy of Sciences.

\section{References}

[1] Lezec H J, Degiron A, Devaux B, Linke R A, Martin-Moreno L, Garcia-Vidal F J and Ebbesen T W 2002 Science 297820

[2] Thio T, Pellerin K M, Linke R A, Lezec H J and Ebbesen T W 2001 Opt. Lett. 261972

[3] Martin-Moreno L, Garcia-Vidal F J, Lezec H J, Degiron A and Ebbesen T W 2003 Phys. Rev. Lett. 90167401

[4] Garcia-Vidal F J, Lezec H J, Ebbesen T W and Martin-Moreno L 2003 Phys. Rev. Lett. 90213901

[5] Yu L-B, Lin D-Z, Chen Y-C, Chang Y-C, Huang K-T, Liaw J-W, Yeh J-T, Liu J-M, Yeh C-S and Lee C-K 2005 Phys. Rev. B 71 041405(R)

[6] Lockyear M J, Hibbins A P, Sambles J R and Lawrence C R 2004 Appl. Phys. Lett. 842040

[7] Akarca-Biyikli S S, Bulu I and Ozbay E 2004 Appl. Phys. Lett. 851098

[8] Caglayan H, Bulu I and Ozbay E 2005 Opt. Express 131666

[9] Kramper P, Agio M, Soukoulis C M, Birner A, Muller F," Wehrspohn R B, Gosele U and Sandoghdar V 2004" Phys. Rev. Lett. 92113903

[10] Moreno E, Garc' 1a-Vidal F J and Mart'ın-Moreno L 2004 Phys. Rev. B 69 121402(R)

[11] Joannopoulos J D, Meade R D and Winn J N 1995 Photonic Crystals: Molding the Flow of Light (Princeton, NJ: Princeton University Press)

[12] Bulu I, Caglayan H and Ozbay E 2005 Opt. Lett. 303078 [13] Morrison S K and Kivshar Y S 2005 Appl. Phys. Lett. 86081110

[14] Tang D, Chen L and Ding W 2006 Appl. Phys. Lett. 89131120

[15] Chen C-C, Pertsch T, Iliew R, Lederer F, Tunnermann A 2006

Opt. Express 142423

[16] Zhu Z H, Ye W M, Ji J R, Yuan X D and Zen C 2007 Appl. Phys. B 86327

[17] Bulu I, Caglayan H and Ozbay E 2003 Appl. Phys. Lett. 83 3263

[18] Caglayan H, Bulu I and Ozbay E 2005 Opt. Express 137645 [19] Mekis A, Chen J C, Kurland I, Fan S, Villeneuve P R and Joannopoulos J D 1996 Phys. Rev. Lett. 773787

[20] Bayindir M, Ozbay E, Temelkuran B, Sigalas M M, Soukoulis C M, Biswas R and Ho K M 2001 Phys. Rev. B $\mathbf{6 3} 081107$

[21] Li Z, Aydin K and Ozbay E 2007 Appl. Phys. Lett. 91121105

[22] Yee K 1966 IEEE Trans. Antennas Propag. 14302

[23] Berenger J-P 1994 J. Comput. Phys. 114185

[24] Moosburger J, Kamp M, Forchel A, Olivier S, Benisty H, Weisbuch C and Oesterle U 2001 Appl. Phys. Lett. 793579

[25] Jensen J S and Sigmund O 2005 J. Opt. Soc. Am. B 221191
[26] Momeni B and Adibi A 2005 Appl. Phys. Lett. 87171104 